Classification

Physics Abstracts

02.50.-r - 07.05.Pj-07.05. $\mathrm{Tp}-68.35 . \mathrm{Ct}$

\title{
Modeling Soil Surface Roughness by Boolean Random Functions
}

\author{
Joël Chadœuf $\left({ }^{1}\right)$, Michel Goulard $\left({ }^{2}\right)$ and Laurent Garcia-Sanchez $\left({ }^{3}\right)$ \\ ( $\left.{ }^{1}\right)$ INRA, Laboratoire de Biométrie, Domaine St Paul, 84914 Avignon Cedex 9, France \\ $\left({ }^{2}\right)$ INRA, Laboratoire de Biométrie, 31326 Castanet-Tolosan, France \\ $\left({ }^{3}\right)$ INRA, Laboratoire de Science du Sol, 84914 Avignon Cedex 9, France
}

\begin{abstract}
Résumé. - Nous présentons dans ce papier une modélisation de la rugosité de la surface du sol par fonctions aléatoires booléennes. Cette classe de fonctions offre l'intérêt de pouvoir intégrer naturellement l'aspect motteux du sol via le choix de fonctions primaires. D'un point de vue statistique, on dispose facilement des lois de distribution des hauteurs, ce qui ouvre la voie à des méthodes classiques d'estimation et de modélisation. Nous présentons ici le cas de l'estimation paramétrique et non-paramétrique de ces modèles, ainsi qu'une modélisation de la non-stationnarité due au travail du sol.
\end{abstract}

\begin{abstract}
In this paper the modeling of soil surface roughness by Boolean random functions is presented. The interest of this class of functions is to be able to model explicitly the cloddy aspect of the soil through the use of primary functions. From a statistical point of view, the height distribution function for such models is easily got, so that classical estimation and modeling methods are available. The case of parametric and non-parametric estimation of the intensity function, and the case of the modeling of the non-stationarity induced by tilling are presented.
\end{abstract}

\section{Introduction}

Soil surface roughness plays a crucial part in controlling depressional storage capacity of surface soils and the distribution of runoff. Huang and Bradford [1] emphasized the necessity to perfect microrelief description models in order to improve depressional storage models. Two main approaches to describe these random surfaces have been conducted in the literature. The first one models the soil surface as a Gaussian-Markov surface [1], characterizing the surface by its covariance function. The second one emphasizes the fractal aspect of the surface. Burrough [2] simulated such a surface by a fractional Brownian function. In fact, for such surfaces self-similarity applies only to the size range of clods and aggregates [3]. So, assuming that the soil surface can be described as an agglomerate of clods and aggregates, Boolean random functions are used [4-6] to model surface roughness. The data sets will be described in a first part. The second part will present the Boolean random function used. The third part is concerned by parametric estimation of the model, the fourth by its non-parametric counterpart. In a last part is presented the modeling of a non-stationarity due to tilling. 


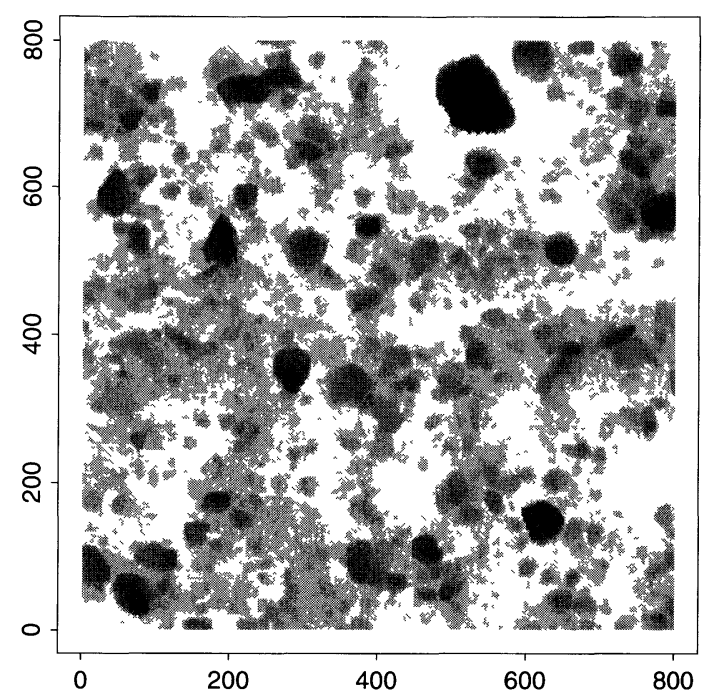

a)

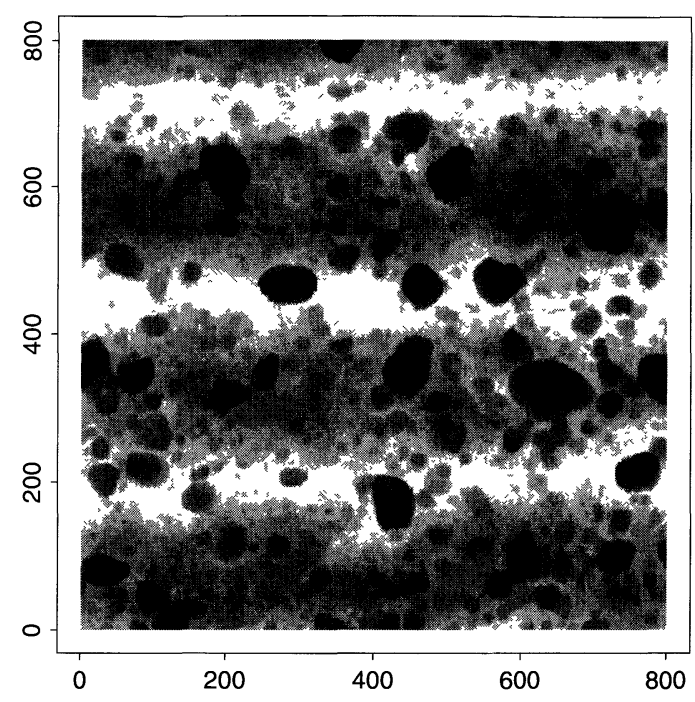

b)

Fig. 1. - Grey-level image of the two soils observed in the first set of experiments. The darker the point the higher the height. a) Isotropic stationary soil sample. b) Sample where the tilling non-stationarity has been left.

\section{The data sets}

Two sets of experiments were conducted. The objective of the first set was to obtain a detailed three-dimensional description of the relief of a tilled surface. The soil was cloddy with a modal clod size of about $40 \mathrm{~mm}$ after tilling. Two square patches, $0.8 \mathrm{~m} \times 0.8 \mathrm{~m}$, were selected and their three-dimensional relief was plotted by stereophotogrametry. For each patch, the output is a square grid of $400 \times 400$ roughness-height values. Horizontal resolution is $2 \mathrm{~mm}$. Figure 1 presents these two observed soils, for one the long range drift due to tilling was removed, whereas for the second one it has been kept.

The second set of experiments was conducted to provide a set of roughness profiles for a soil affected by rain. Four series of roughness-height profiles were recorded at the initial stage and under three successive irrigations $(0,30,64,100 \mathrm{~mm})$. Each series includes 24 roughness-height profiles measured with a laser profile meter [3]. Each profile is $1 \mathrm{~m}$ long and the horizontal step is $2 \mathrm{~mm}$.

\section{The used Boolean random function}

A general definition of Boolean random functions can be found in [6]. The model used here is based on:

- a given primary function $f$ from $\mathbb{I}^{2}$ to $\mathbb{R}$ positive, symmetric around $(0,0)$, convex and satisfying $f(x, y)=0$ if $x^{2}+y^{2}>1$. Let $f_{0}$ such that $f(x, y)=f_{0}\left(\sqrt{x^{2}+y^{2}}\right)$.

- a Poisson point process $X$ on $\mathbb{R}^{2} \times \mathbb{R}^{+}$of intensity $a(z)$, that is, for $\mathbf{V} \subset \mathbb{R}^{2} \times \mathbb{R}^{+}$, the number of points of $\mathrm{X}$ falling in $\mathrm{V}$ is Poisson with parameter $\int_{(x, y, z) \in \mathrm{V}} a(z) \mathrm{d} x \mathrm{~d} y \mathrm{~d} z$

To each point $\left(x_{i}, y_{i}, z_{i}\right) \in \mathrm{X}$ is attached a function $f_{i}(x, y)=z_{i} f\left(\left(x+x_{\imath}\right) / z_{\imath},\left(y+y_{\imath}\right) / z_{\imath}\right)$. The Boolean random function $Z(x, y)$ is defined as the supremum of all functions $f_{\imath}$. The upper right 


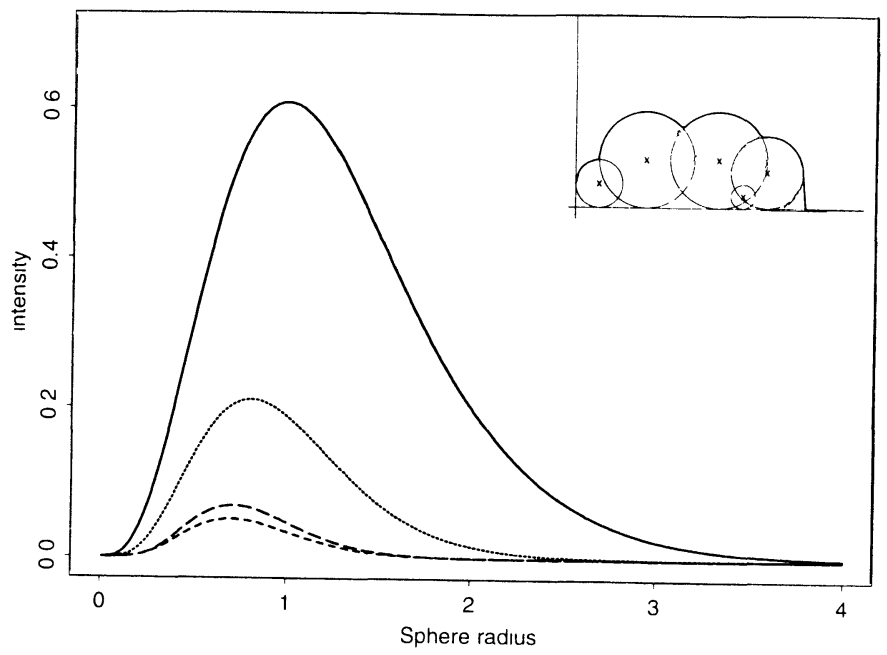

Fig. 2. - Estimated Boolean random function intensities for the successive 0, 30, 64 and $100 \mathrm{~mm}$ cumulative rainfall. The highest the curve, the less the cumulative rainfall. Upper right corner: example of a Boolean random function on the line with the umbra of the sphere as primary grain.

corner of Figure 2 presents the case of a Boolean random function on the line, where $f$ is the umbra of a disc. In the following, the field $Z$ observed at the nodes of a regular grid $\mathrm{G}$, or at points regularly located on a line will be considered. For such a random field, one gets classically

$$
P\left(Z_{(x, y)} \leq h\right)=\exp \left\{-\pi \int_{h / f_{0}(0)}^{\infty}\left(f_{0}^{-1}(h / z)\right)^{2} z^{2} a(z) \mathrm{d} z\right\} .
$$

Moreover, for sufficiently decreasing functions $a(z)$, i.e., as soon as $\int_{r}^{\infty} z^{2} a(z) \mathrm{d} z \rightarrow 0$ when $r \rightarrow \infty$, a mixing property is obtained [7]: let $\mathrm{A}$ and $\mathrm{B}$ be finite sets on the grid $\mathrm{G}, n(\mathrm{~A})$ and $n(\mathrm{~B})$ the number of points of $\mathrm{A}$ and $\mathrm{B}$. If $\mathrm{U}$ and $\mathrm{V}$ are two events defined on $\mathrm{A}$ and $\mathrm{B}$, then, for $r>d(\mathrm{~A}, \mathrm{~B}) / 2$,

$$
|P(\mathrm{U} \cap \mathrm{V})-P(\mathrm{U}) P(\mathrm{~V})| \leq C \min (n(\mathrm{~A}), n(\mathrm{~B})) \int_{r}^{\infty} z^{2} a(z) \mathrm{d} z
$$

i.e., events on A and B become independent when the distance between A and B increases. Therefore, estimation based on the observed heights of the random field is possible using formula (1), while formula (2) will ensure the convergence of the estimators using the mixing property of the field.

\section{Parametric Estimation}

Let us consider the case where $f$ is the umbra of a sphere of radius 1 as in the above example and suppose that $a(z)$ is known up to a given number of parameters. In [7] $a(z)=\alpha z^{\beta} \exp (\lambda z)$ was considered. $\alpha$ corresponds to a scaling factor, $\beta$ and $\lambda(\lambda<0)$ are shape parameters. $\lambda$ controls the decrease to zero as $r$ grows while $\beta$ controls the number of small spheres. From formula (1) one derives

$$
P\left(Z_{(x, y)} \leq h\right)=\exp \left\{-\pi \int_{h / 2}^{h} h(2 z-h) a(z) \mathrm{d} z-\pi \int_{h}^{\infty} z^{2} a(z) \mathrm{d} z\right\}
$$


Table I. - Estimated parameters of the first model for the second set of experiments.

\begin{tabular}{|c|c|c|c|}
\hline $\begin{array}{c}\text { Cumulative } \\
\text { rainfall }(\mathrm{mm})\end{array}$ & $\alpha\left(\mathrm{cm}^{-2}\right)$ & $\beta\left(\mathrm{cm}^{-1}\right)$ & $\lambda\left(\mathrm{cm}^{-1}\right)$ \\
\hline 0 & 20.35 & 3.47 & -3.51 \\
30 & 49.62 & 4.49 & -5.57 \\
64 & 108.07 & 5.66 & -8.06 \\
100 & 249.65 & 6.12 & -8.59 \\
\hline
\end{tabular}

and the set of parameters $(\alpha, \beta, \lambda)$ is estimated by a maximum of pseudo-likelihood method: the estimator is defined as the set of parameters maximizing the product of the probabilities of the observed heights.

Table I presents the sets of estimated parameters for the second experiment [8], while Figure 2 presents the estimated intensities corresponding to the successive rainfalls. The estimated values were highly sensitive to cumulative rainfall. Parameters $\alpha$ and $\beta$ increased while $\lambda$ decreased corresponding to a fewer number of big clods when the cumulative rainfall increases. The mode of the intensity function decreased corresponding to the reduction of the clod size with the rainfalls.

\section{Non-Parametric Estimation of the Intensity}

The preceding method requires knowledge of both the shape of the primary grain and the intensity function, even if though it is imposed by a finite number of parameters. Such a choice is not easy, more particularly for the intensity function. However, let us consider the case where the primary grain is a semi-sphere of radius 1 lying on the horizontal plane $z=0$. Then, one obtains from equation (1):

$$
P(h)=P\left(Z_{(x, y)} \leq h\right)=\exp \left\{-\int_{h}^{\infty} \pi\left(z^{2}-h^{2}\right) a(z) \mathrm{d} z\right\}
$$

from which one easily gets $A(r)=\int_{r}^{\infty} a(z) \mathrm{d} z=\frac{p(r)}{2 \pi r P(r)}$ and $a(z)=\frac{p(z)}{2 \pi z^{2} P(z)}+\frac{p(z)^{2}}{2 \pi z P(z)^{2}}-\frac{p^{(1)}(z)}{2 \pi z P(z)}$ where $P$ is the cumulative distribution function of heights, $p$ the density function of heights and $p^{(1)}$ its derivative.

Estimators of $A(r)$ and $a(z)$ are derived directly from these formulae by replacing $P$ by the empirical distribution of heights, $\hat{P}(r)=\frac{1}{n^{2}} \sum_{i, j=1}^{n} 1_{\left\{Z_{2, j} \leq r\right\}}, p$ and $p^{(1)}$ by their kernel estimators [9] calculated on the observed image [10]. For example, using the kernel $K(u)=\frac{3}{4}\left(1-u^{2}\right)$ if $|u|<1, K(u)=0$ otherwise, one gets: $\hat{p}(r)=\frac{1}{\omega n^{2}} \sum_{i, j=1}^{n} K\left(\frac{r-Z_{, .,}}{\omega}\right)$ and $\hat{p}^{(1)}(r)=\frac{1}{\omega^{2} n^{2}} \sum_{i, j=1}^{n} K^{(1)}\left(\frac{r-Z_{i, j}}{\omega}\right)$ where $\omega$ is the width of the smoothing window whose value tends to 0 with $n$. Replacing $P, p$ and $p^{(1)}$ by these estimators, one gets non-parametric estimators of $A$ and $a$, asymptotically unbiased and of known asymptotic variance under very mild conditions [10]. Figure 3 presents confidence bands of $A(r)$ and $a(z)$ using such estimators for the two extreme cases of the second data set (initial stage and after $100 \mathrm{~mm}$ cumulative rainfall). 


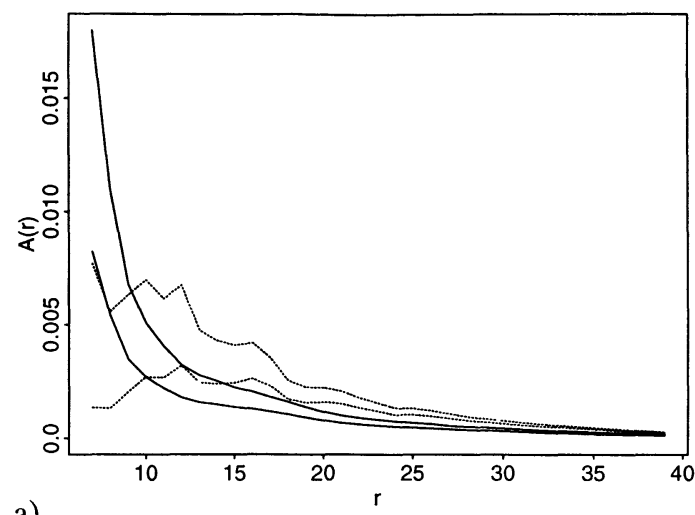

a)

Fig. 3. - Non parametric confidence bounds of the cumulative Boolean random function intensities $A(r)$ (a) and of the Boolean random function intensities $a(r)$ (b) for the $0 \mathrm{~mm}$ (plain lines) and $100 \mathrm{~mm}$ cumulative rainfalls(dotted lines).

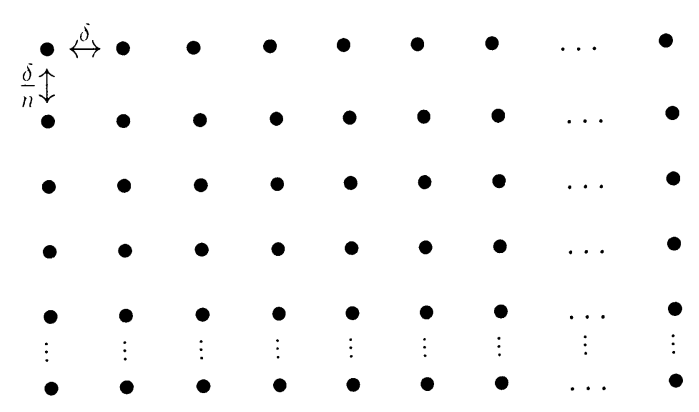

Fig. 4. - Sampling scheme used in the case of non-stationarity parallel to the $x$-axis. Distance between horizontal lines $\delta / n$ with $n \rightarrow 0$. Distance between consecutive points of measure on an horizontal line $\delta$.

\section{Modeling Non-Stationarity}

In the preceding sections, the surface was considered stationary. However, tilling induces a onedimensional non-stationarity (see Fig. 1b), by creating furrows. So the soil surface may be modeled as the sum of a determinist term describing the furrow and a random Boolean function describing the random roughness: $Z(x, y)=G(x)+Z_{0}(x, y)$. Moreover, at a lower scale, tilling may induce a non-stationarity on the roughness itself. Let us consider as before the case of a semi-sphere as primary grain, and suppose that the roughness is bounded. This last effect can be easily modeled in the intensity function through a multiplicative model: $a(x, z)=\lambda(x) a_{0}(z)$, where $a_{0}$ and $\lambda$ are continuously derivable functions and satisfy: $\sup \left\{z ; a_{0}(z)>0\right\}=R<\infty$, $\left|\frac{1}{V} \int_{0}^{V} \lambda(v) \mathrm{d} v-1\right| \leq C / V, 0<l<\lambda(x)<L<\infty$ and $\left|\frac{\mathrm{d} \lambda(x)}{\mathrm{d} x}\right| \leq L^{\prime}$. For that model, denotirg $A(r)=\int_{r}^{\infty} a(z) \mathrm{d} z$, the probability of observing a height less than $h+G(x)$ at coordinates $(x, y)$ is:

$$
\log P\left(Z_{x, y} \leq h+G(x)\right)=-\int_{v=-\infty}^{\infty} \int_{w=-\infty}^{\infty} \lambda(x+v) A\left(\sqrt{v^{2}+w^{2}+h^{2}}\right) \mathrm{d} v \mathrm{~d} w
$$




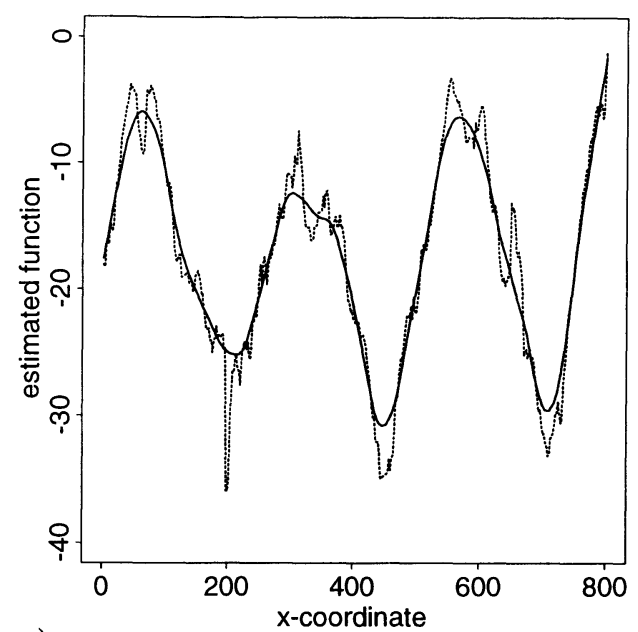

a)

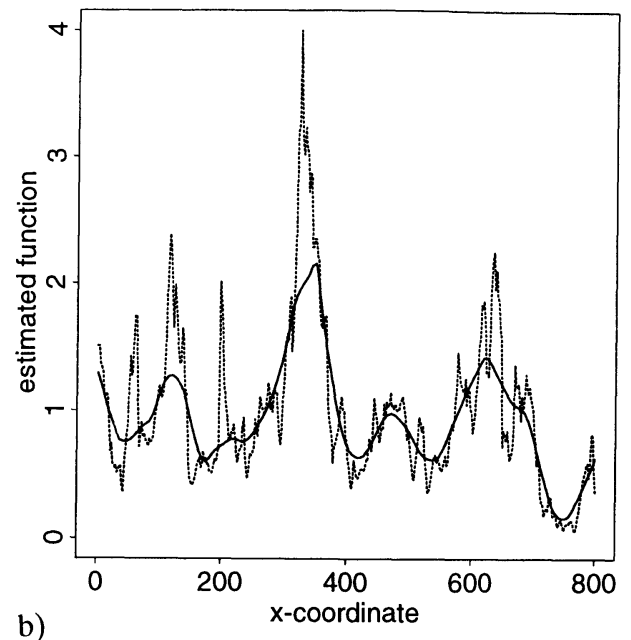

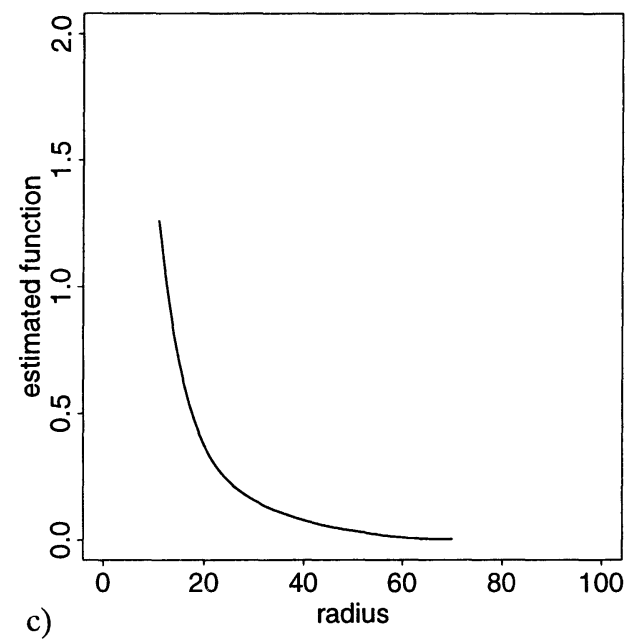

Fig. 5. - Estimated determinist drift $G(x)$ (curve a) component of the intensity $\lambda(x)$ (curve b) and of $V(r)$ (curve c) (see text) for the second sample of the first set of experiments. Plain lines of a) and b): local robust smoothing of the estimated curves drawn in dotted lines using lowess procedure with parameter 0.1 (see [12] for details).

Let us suppose that the process is sampled along $n^{2}$ lines distant of $\delta / n$ parallel to the tilling direction along the $x$-direction, the distance between two successive points on a line being $\delta$ (see Fig. 4), a sampling scheme easily performed by 2D laser profile meters. Let $h_{n} \rightarrow R$, then, if $i_{x}$ is the integer part of $\delta x$, and $\hat{P}_{i}(h)$ is the empirical distribution function of heights estimated along line $i$,

$$
\hat{G}(x)=\inf _{j=1 . . n} Z_{i_{x}, j}, \quad \hat{V}(h)=\frac{1}{2 \pi n^{2}} \sum_{1}^{n^{2}} \log \left(\hat{P}_{i}(h+\hat{G}(i \delta / n)), \quad \hat{\lambda}(x)=\frac{\log \left(\hat{P}_{i_{x}}\left(h_{n}+\hat{G}\left(i_{x}\right)\right)\right)}{\hat{V}\left(h_{n}\right)}\right.
$$

are asymptotically unbiased consistent estimators of $G(x), V(h)=\int_{h}^{\infty} u A(u) \mathrm{d} u$ and $\lambda(x)[11]$. 
Figure 5 presents the estimated curves corresponding to the second sample of the first set of experiments. The tilling effect can be noticed on both curves $\hat{G}(x)$ and $\hat{\lambda}(x)$. The estimated deterministic drift $\hat{G}(x)$ corresponds to the global effect which can be already observed in Figure 1b. It can be noticed on the curve $\hat{\lambda}(x)$, the estimated spatial component of the intensity, an effect which was difficult to perceive on the initial image: a non-stationary effect of the roughness component of the soil surface, with a period roughly half of that of the determinist drift.

\section{Conclusion}

Boolean random functions can be a very useful tool to study surface roughness. They lead to a good description of the clods and aggregates through the use of a primary grain. Moreover the intensity function can be analyzed in the same way as a granulometry function.

The definition of this model is sufficiently large to allow an easy introduction of various assumptions on the process, as it can be seen for non-stationnarity due to tilling.

At the moment the main restriction is that their estimation has been performed conditionally to the shape of the primary grain. The choice of a specific shape is always somewhat arbitrary. Further work should try to get rid of this assumption.

\section{References}

[1] Huang C. and Bradford J.M., Depressional storage for Markov-Gaussian surfaces, Water Res. Res. 26 (1990) 2235-2242.

[2] Burrough P.A., Multiscale sources of spatial variation in soil. II. A non-Brownian fractal model and its application in soil survey, J. Soil Sci. 34 (1983) 599-620.

[3] Bertuzzi P., Rauws G. and Courault D., Testing roughness indices to estimate soil surface roughness changes due to simulated rainfall, Soil Tillage Res. 17 (1990) 87-99.

[4] Jeulin D. and Jeulin P., Synthesis of rough surfaces of random morphological functions, Stereol. Iugosl. (suppl. 1) 3 (1981) 239-246.

[5] Jeulin D., Anisotropic Rough Surface Modelling by Random Morphological Functions, Acta Stereol. 6/I (1987) 183-189.

[6] Serra J., Boolean random functions J. Microsc. 156 (1989) 41-63.

[7] Chadœuf J., Monestiez P., Bertuzzi P. and Stengel P., Parameter estimation in a Boolean model of rough surface: application to soil surfaces, Acta Stereol. 8 (1989) 635-640.

[8] Bertuzzi P., Garcia-Sanchez J., Chadœuf J., Guerif J. and Goulard M., Modelling surface roughness by a Boolean approach, Eur. J. Soil Sci. 46 (1995) 215-220.

[9] Silverman B.W., Density Estimation for Statistics and Data Analysis (Chapman and Hall, London, 1986).

[10] Goulard M., Chadœuf J. and Bertuzzi P., Random Boolean functions : non-parametric estimation of the intensity. Application to soil surface roughness, Statistics 25 (1994) 123-136.

[11] Chadœuf J., Elguero E., Guerif G., Goulard M. and Bertuzzi P. Estimation of one dimensional nonstationarity in random surfaces using Boolean random functions, Technical Report, INRA, Laboratoire de Biométrie d'Avignon (1992).

[12] Chambers J.M., Cleveland W.S., Kleiner B. and Tukey P.A., Graphical methods for data analysis (Wadsworth \& Brooks, Pacific Groove, 1983). 